\title{
Autophagy is required for zebrafish caudal fin regeneration
}

\author{
M Varga ${ }^{1}$, M Sass ${ }^{2}$, D Papp ${ }^{1}$, K Takács-Vellai ${ }^{1}$, J Kobolak ${ }^{3}$, A Dinnyés ${ }^{3}$, DJ Klionsky ${ }^{\star, 4}$ and T Vellai ${ }^{1}$
}

Regeneration is the ability of multicellular organisms to replace damaged tissues and regrow lost body parts. This process relies on cell fate transformation that involves changes in gene expression as well as in the composition of the cytoplasmic compartment, and exhibits a characteristic age-related decline. Here, we present evidence that genetic and pharmacological inhibition of autophagy - a lysosome-mediated self-degradation process of eukaryotic cells, which has been implicated in extensive cellular remodelling and aging - impairs the regeneration of amputated caudal fins in the zebrafish (Danio rerio). Thus, autophagy is required for injury-induced tissue renewal. We further show that upregulation of autophagy in the regeneration zone occurs downstream of mitogen-activated protein kinase/extracellular signal-regulated kinase signalling to protect cells from undergoing apoptosis and enable cytosolic restructuring underlying terminal cell fate determination. This novel cellular function of the autophagic process in regeneration implies that the role of cellular self-digestion in differentiation and tissue patterning is more fundamental than previously thought.

Cell Death and Differentiation (2014) 21, 547-556; doi:10.1038/cdd.2013.175; published online 6 December 2013

Understanding the mechanism and regulation of tissue regeneration is a fundamental and fascinating problem in biology, with significant medical implications. ${ }^{1}$ Intensive research in the past decade provided evidence that animals have evolved three main mechanisms to restore damaged tissues: the reactivation of resident, multipotent adult stem cells, the dedifferentiation of mature somatic cells near the plane of injury into dividing unipotent progenitors, and, in specific instances, transdifferentiation, during which terminally specialized cells switch lineages to adopt fates different from the original ones. ${ }^{2-4}$ Zebrafish has emerged as a powerful vertebrate genetic model to study regeneration. It can replace up to one-fifth of its heart ventricle after injury, ${ }^{5,6}$ and has a seemingly unlimited potential to regrow its caudal fin after amputation. ${ }^{7,8}$

The zebrafish caudal fin has become a useful proxy to examine the paradigm of appendage regeneration. Similar to renewal of amphibian limbs, caudal fin regeneration starts from a highly proliferative tissue, the blastema, which is formed distal from the site of amputation via dedifferentiation and generates almost exclusively cells of the parent cell's type ${ }^{9-12}$ For example, dedifferentiating cells derived from mature osteoblasts in the zebrafish fin give rise to osteoblasts only. Of note, recent evidence suggests the existence of a putative second source of osteoblast precursors. ${ }^{13}$ In the case of the zebrafish fin, most major signalling pathways have been implicated in the regenerative process, ${ }^{14-16}$ including Fgf (fibroblast growth factor) signalling that occupies a prominent, upstream position in the regulatory network driving blastema formation. ${ }^{17,18}$

Cell fate transformation ('reprogramming') underlying dedifferentiation occurs in both the nucleus (changes in global gene expression) and the cytoplasm (changes in cytoplasmic constituents). Previous studies described profound alterations in the cellular composition of dedifferentiating osteoblasts. ${ }^{10}$ Extensive remodelling of the cytoplasmic compartment involves the synthesis of new proteins and, in parallel, the degradation of existing ones. The latter process occurs predominantly by autophagy ('cellular self-eating'), during which parts of the cytoplasm are delivered to the lysosome for degradation by hydrolytic enzymes, in particular proteases. ${ }^{19}$ Consistent with this primordial role of autophagy in cellular remodelling, autophagic degradation has been implicated in diverse developmental and differentiation processes in divergent animal taxa ranging from nematodes to mammals. ${ }^{20,21}$

Here we tested whether cytoplasmic reorganisation enabling dedifferentiation that precedes caudal fin regeneration in zebrafish requires autophagy. We provide evidence that autophagy is activated in the blastema during fin regeneration, and that an elevated level of autophagic activity is indispensable for fin regrowth. According to our data, inactivation of autophagy stimulates mesenchymal blastema cells to undergo apoptosis, and causes defects in the

\footnotetext{
${ }^{1}$ Department of Genetics, Eötvös Loránd University, Budapest, Hungary; ${ }^{2}$ Department of Anatomy, Cell and Developmental Biology, Eötvös Loránd University, Budapest, Hungary; ${ }^{3}$ BioTalentum Ltd., H-2100, Gödöllö, Hungary and ${ }^{4}$ Life Sciences Institute, University of Michigan, Ann Arbor, MI, USA

*Corresponding author: DJ Klionsky, Life Sciences Institute, University of Michigan, 210 Washtenaw Avenue, Ann Arbor, Ml 48109-2216, USA. Tel: +1 1734 615 6556; Fax: +1 1734647 9702; E-mail: klionsky@umich.edu

Keywords: autophagy; regeneration; zebrafish; differentiation; cell death

Abbreviations: Atg, autophagy-related; DNRas, dominant-negative form of Ras; Dpa, days post amputation; Erk, extracellular signal-regulated kinase; Fgf, fibroblast growth factor; GFP, green fluorescent protein; GFP-LC3, GFP fused at the N terminus to LC3; Hpi, hours post injection; Lc3, microtubule-associated protein 1 light chain 3; Mapk, mitogen-activated protein kinase; mkp3, mitogen-activated protein kinase phosphatase 3; MO, synthetic antisense morpholino oligonucleotide; PBST, phosphate-buffered saline containing $0.1 \%$ Tween-20; PFA, paraformaldehyde; TUNEL, terminal deoxynucleotidyl transferase-mediated dUTP nick end labelling Received 4.3.13; revised 29.10.13; accepted 31.10.13; Edited by E Baehrecke; published online 06.12.13
} 
expression of differentiation markers characterising terminal cell fate determination. We conclude that autophagy is required for survival and differentiation of cells in this model of tissue regeneration, and may have an essential role in various regeneration processes such as wound healing or the generation of new neurons to combat neurodegenerative diseases in humans.

\section{Results}

Autophagy is upregulated in the blastema during zebrafish caudal fin regeneration. Dramatic changes in osteoblast morphology during dedifferentiation ${ }^{10}$ suggested the possibility that some regulated process of cytoplasm reorganisation is involved in zebrafish fin regeneration. Our aim was to assess whether this process involves autophagy, a highly conserved pathway of cellular self-degradation. ${ }^{19,22}$ To test this hypothesis, we amputated the caudal fins of adult transgenic zebrafish carrying a green fluorescent protein-microtubule-associated protein 1 light chain 3 isoform (GFP-Lc3) reporter. ${ }^{23}$ Lc3 is a zebrafish orthologue of the yeast Atg8 (autophagy-related 8) protein and mammalian MAP1LC3 (microtubule-associated protein 1 light chain 3) proteins, and the Atg8/LC3 family of proteins is widely used to detect macroautophagic structures (e.g., autophagosomes and autolysosomes) in various species. ${ }^{22,24}$ In zebrafish that underwent amputation, expression intensity of the GFP-LC3 reporter was markedly upregulated in the fin, proximal to the injury plane and in the blastema 2 days post amputation (dpa), then gradually decreased to nearly normal levels during the rest of the regeneration process (Figures 1a-c). During this period, transgene expression could be observed in newly differentiated cells, with the highest levels in the proliferating zone, close to the tail tip. As a

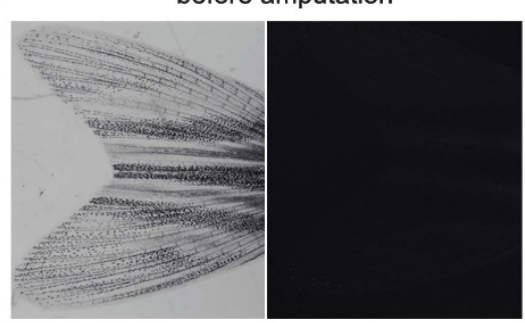

b

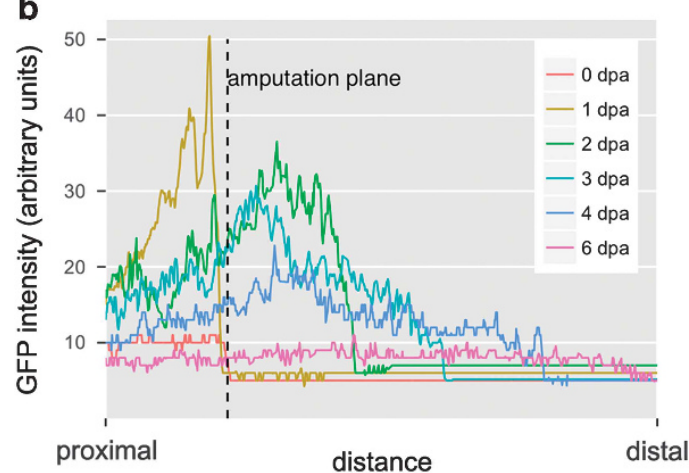

$0 \mathrm{dpa}$
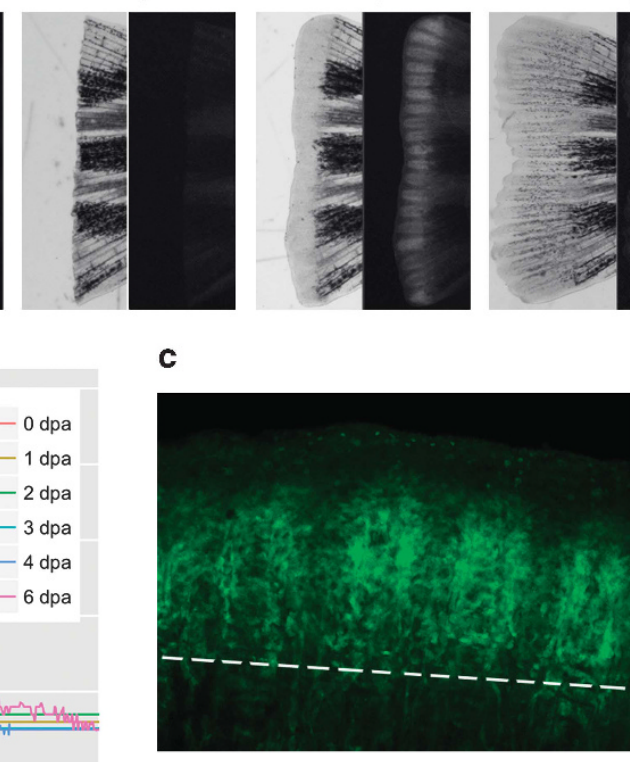

$4 \mathrm{dpa}$

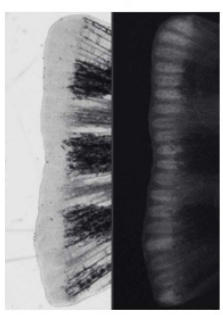

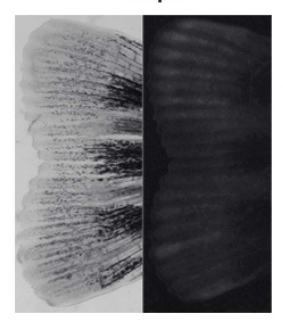

6 dpa
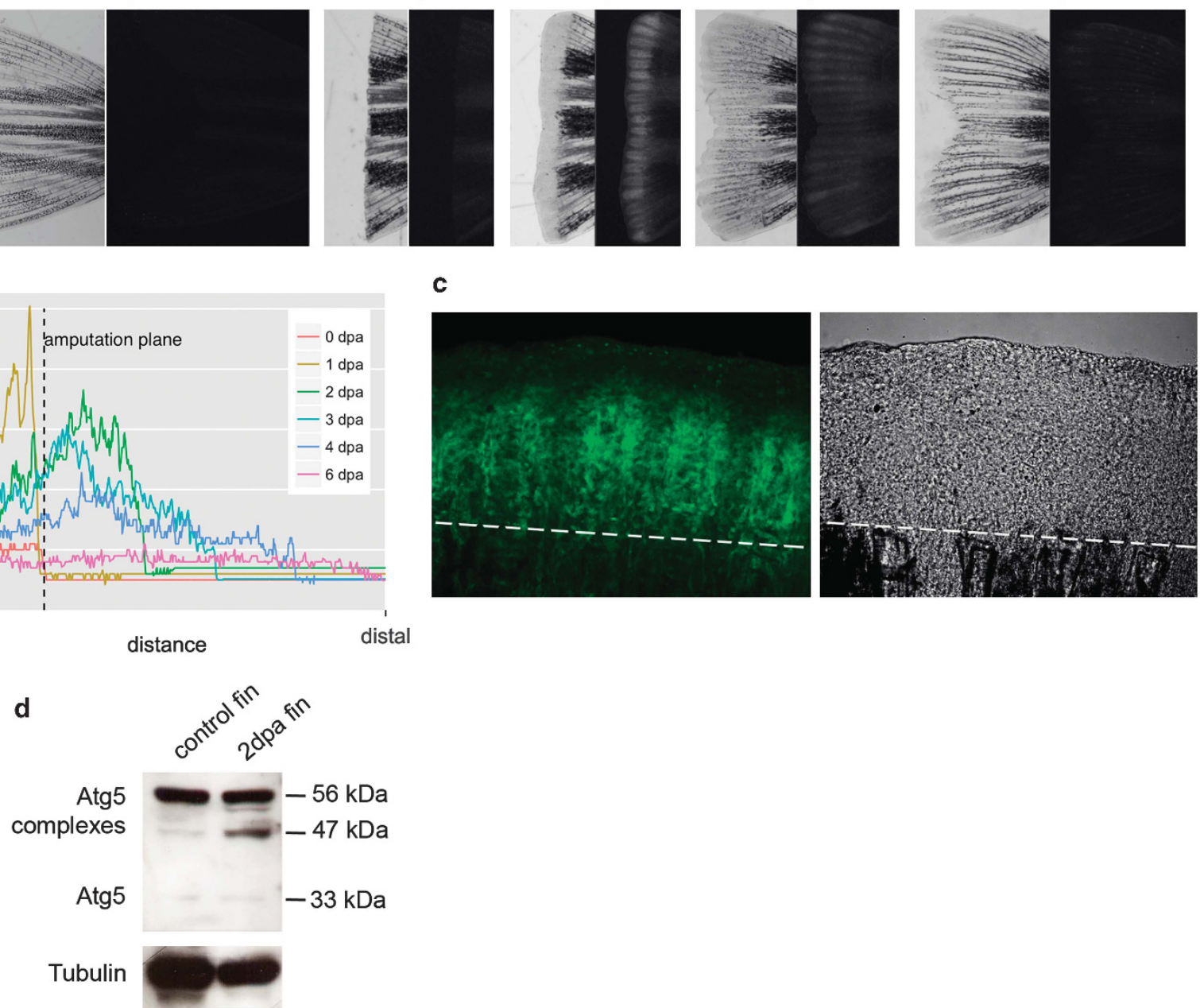

Figure 1 Autophagy is upregulated during zebrafish caudal fin regeneration. (a) Intense accumulation of the autophagy marker Lc3/Atg8 in the blastema tissue. Expression of GFP-LC3 in a zebrafish caudal fin before, at the time of amputation and 2, 4 and 6 days thereafter (dpa). Left: light microscopy images; right: the corresponding fluorescent images. (b) Quantification of fluorescence intensity for a representative fin ray during regeneration. The plane of amputation is kept fixed, and fluorescence is shown relative to this position. Intensity was plotted for the same fin ray over the regeneration period using ImageJ. (c) Confocal images of a 2 dpa GFP-Lc3 blastema. Some GFP-positive foci labelling autophagic elements are interconnected by filamentous structures. The white broken line indicates the amputation plane. (d) Western blots of intact and regenerating tails indicate a strong increase in the amount of complexed Atg5. On average, the amount of Atg5 complexes is roughly $60 \%$ higher in 2 dpa regenerates than in control tissues, and in regenerating blastemas, we consistently detect a new $\sim 47-\mathrm{kDa}$ complex, besides the more common $\sim 56-\mathrm{kDa}$ one 
these cells are descendants of previously dedifferentiated cells, reporter activity could reflect an increased autophagic activity during the dedifferentiation-redifferentiation process.

Analysis of regenerating tails also showed elevated levels of complexes containing Atg5, a mediator of the lipidation and binding of soluble Lc3 (LC3-I) to the phagophore membrane (Lc3-II), ${ }^{25}$ in the blastema (Figure 1d). Interestingly, independent experiments in regenerating fins repeatedly showed a stark increase in the amount of a $47-\mathrm{kDa}$ complex, which is present only slightly above background levels in intact controls (Figure 1d). The level of different Atg5-containing complexes changed relative to $\gamma$-tubulin (Figure 1d), and thus provided evidence for a selective upregulation of the autophagic process, rather than for a nonselective elevation of protein synthesis. Indeed, a recent proteomic analysis of the regenerating caudal fin provided unequivocal evidence for the differential regulation of dozens of proteins. ${ }^{26}$

Consistent with the idea that the GFP-Lc3 expression data above reflect an increased rate of autophagy during early phases of regeneration, electron microscopy revealed an elevated number of autophagic structures in several cell types of the $2 \mathrm{dpa}$ fins, compared with untreated control tissues (Figures $2 a-g$ ). As revealed by quantification of the autophagic structures (Figure $2 \mathrm{~g}$ ), the increase in autophagic activity in epidermal cells, in osteocytes and in pigment cells detected at the ultrastructural level mirrors the reporter-transgene activity observed in the GFP-LC3 line. The number of autophagic structures was most abundant after 2 days of amputation and decreased by 4 dpa. Together, these results imply a massive upregulation of autophagy in the regenerating blastema tissue.

Autophagy is required for caudal fin regeneration. To test whether autophagy is necessary for caudal fin regeneration, we used several strategies to block the process. First, we injected synthetic antisense morpholino oligonucleotide (MO) targeting the translation site of $\operatorname{atg} 5$ (Atg5MO) into the dorsal part of the fin stump at 2 dpa (Figures $3 a$ and $b^{\prime}$ ). Depletion of Atg5 was able to effectively block regeneration in the injected fin area, and even induce the degeneration of the existing blastema (Figures $3 a-c$ ). As these effects are significantly different from those observed when MilliQ water or a standard control MO were used for injections (Figure 2e), and the same morpholino caused specific defects during embryonic development (Supplementary Figure S1), similar to phenotypes described before for an overlapping Atg5 morpholino, ${ }^{27}$ its antiregenerative effect is not merely due to a general toxicity of the reagent.

To further confirm these results, animals that underwent amputation were also treated with the late-stage autophagy inhibitor bafilomycin $A_{1}$, which blocks both starvation-induced and non-starvation-dependent autophagy. ${ }^{28}$ Bafilomycin $\mathrm{A}_{1}$-treated animals also lost their ability to regenerate their caudal fin (Figures $3 \mathrm{f}-\mathrm{j}$ ). In addition, the treatment provoked a massive activity of GFP-LC3 reporter in the blastema (Figure $3 \mathrm{j}$ ), supporting previous findings that showed an inhibitory effect of bafilomycin $\mathrm{A}_{1}$ on autophagosome-autolyosome fusion, ${ }^{28}$ and further suggesting an important role for autophagy in the regeneration process. Interestingly, a similar phenotype was observed when fish were incubated with chloroquine, another small-molecule inhibitor of the autophagic process (Supplementary Figure S2), suggesting that the effects of bafilomycin $A_{1}$ were not merely due to nonspecific effects of the drug.

Compromised autophagy triggers apoptotic cell death and blocks differentiation in the blastema. As inhibition of autophagy triggers apoptosis in some in vivo models, ${ }^{29,30}$ we monitored whether the reduced regenerative capacity of Atg5MO-injected or bafilomycin $\mathrm{A}_{1}$-treated fin tissues is due, at least in part, to an increase in apoptotic activity. Using TUNEL (terminal deoxynucleotidyl transferase-mediated dUTP nick end labelling) staining that specifically marks fragmented DNA in apoptotic cells, we examined apoptosis in the blastema of Atg5MO-injected dorsal fins and found an increased rate of cell death at $6 \mathrm{~h}$ post injection (hpi), compared with control ventral fins (Figures $4 \mathrm{a}-\mathrm{c}$ ). Quantification of TUNEL-positive cells in autophagy-defective dorsal fin tissues showed elevated levels of cell death compared with control fins (Figure 4c). Increased numbers of apoptotic cells were also observable in bafilomycin $\mathrm{A}_{1}$-treated samples (Figures 4d and e). Atg5-specific morpholino and bafilomycin $A_{1}$ treatments also reduced the proliferative capacity of the blastema, as revealed by anti-phospho-histone 3 staining on 3 dpa regenerates ${ }^{31}$ (Figures $4 \mathrm{f}, \mathrm{g}, \mathrm{I}, \mathrm{m}$ ). These results suggest that impaired autophagy prevents both survival and proliferation of dedifferentiating cells at early stages of the regeneration process.

Furthermore, we found that surviving blastema cells in Atg5MO-treated fin tissues are not able to differentiate properly, as they showed impaired expression of the preosteoblast marker runx $2 b$, normally expressed at the top of the growing fin rays ${ }^{10}$ (Figure $4 \mathrm{~h}$ ). According to our data, runx $2 b$ was completely absent from the regenerative zone of caudal fins in the Atg5MO genetic background (Figure 4i). The expression of the differentiated osteoblast reporter osteocalcin:GFP ${ }^{10}$ was also compromised in Atg5MO-treated animals (Figures 4j and k). Impaired expression of osteocalcin:GFP was also evident upon chronic bafilomycin $A_{1}$ treatment (Figures $4 n-o^{\prime}$ ). Thus, proliferating cells behind the amputation plane in an autophagy-defective background failed to differentiate into mature osteoblasts. We conclude that autophagy promotes both cell survival and proliferation, and mediates differentiation in the regenerative zone during the regrowth of zebrafish caudal fin.

Mapk/Erk signalling is essential for and regulates autophagy during caudal fin regeneration. Fgf signalling has a well-established role in zebrafish fin regeneration, and, according to previous reports, inhibition of Fgf signalling blocks the dedifferentiation and concomitant cytoplasmic reorganisation of osteoblasts. ${ }^{10,18,32}$ As one of the effector (downstream) mechanisms of Fgf signalling is the mitogenactivated protein kinase/extracellular signal-regulated kinase (Mapk/Erk)-mediated signal transduction system, ${ }^{33}$ which has an important role in the regulation of autophagy, ${ }^{34,35}$ we tested whether the impairment of Mapk/Erk influences the regeneration capacity of amputated caudal fins. Using the Mapk/Erk inhibitor U0126, ${ }^{36}$ we observed an almost 

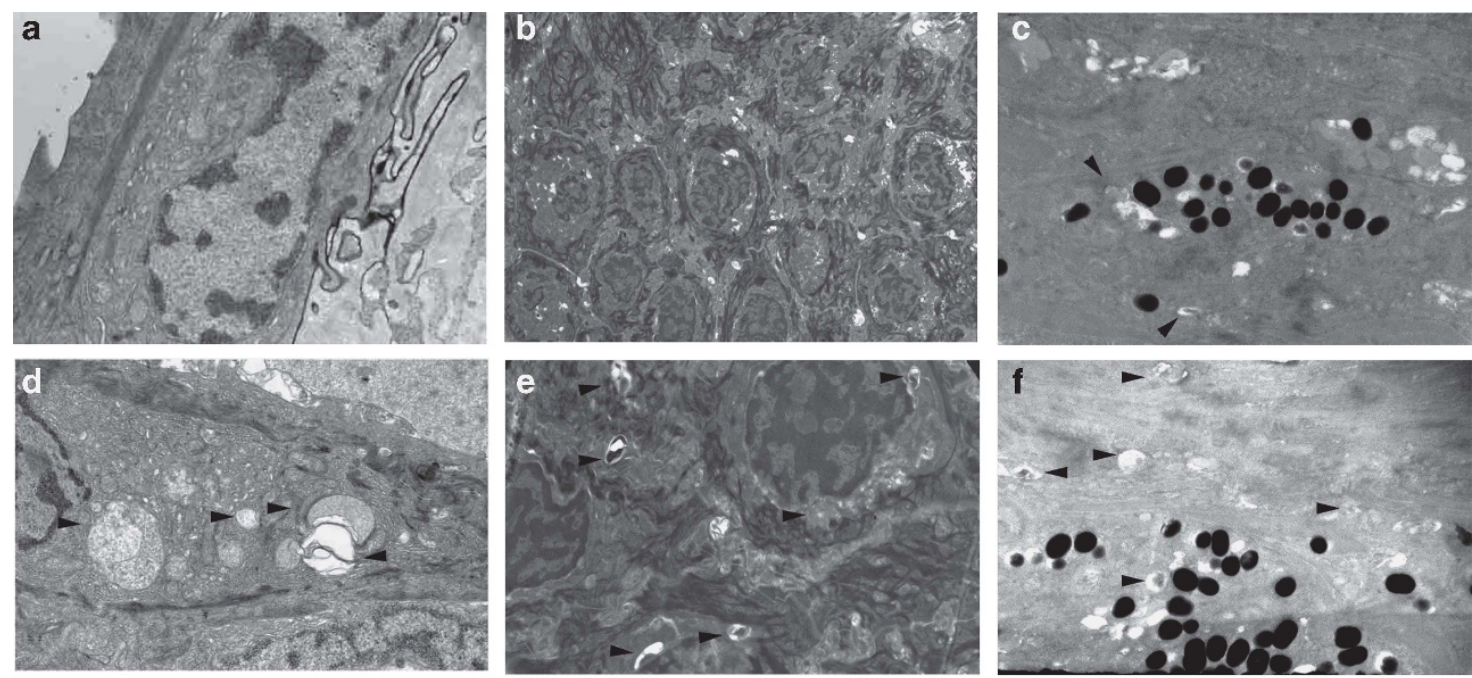

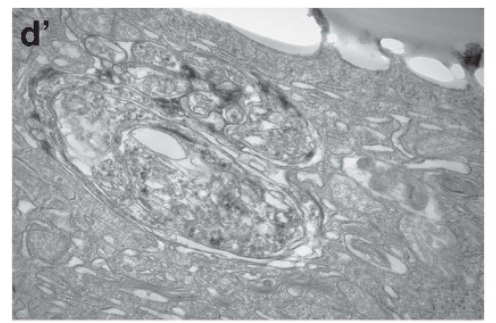

epidermal cells

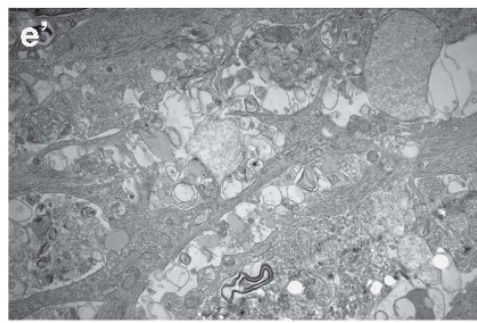

osteocytes

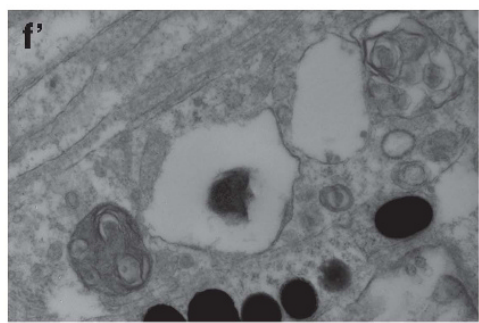

pigment cells

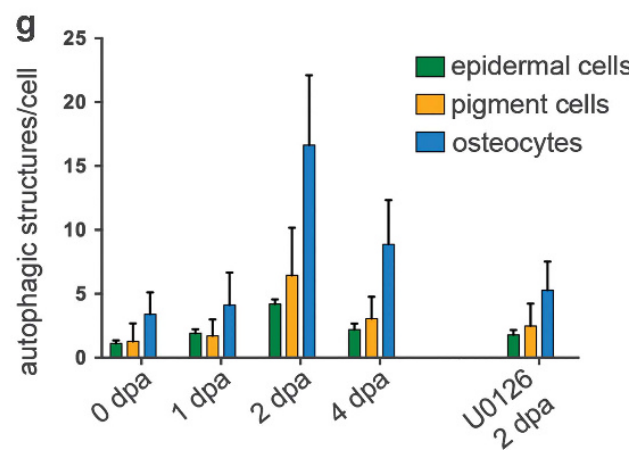

Figure 2 Ultrastructural evidence for an increase in autophagy in the regenerating blastema tissue. Electron microscopy pictures of epidermal cells (a), osteocytes (b) and pigment cells (c) from the tail tip region of control animals show no sign or only low levels of autophagic activity. In the corresponding tissues of the regenerating zone of a 2-dpa fin, elevated numbers of autophagic structures (arrowheads) can be observed $\left(\mathbf{d}-\mathbf{f}^{\prime}\right)$. (g) The quantification of autophagic vesicles in our EM data set shows a temporary increase in the number of these structures, with the maximum observed at $2 \mathrm{dpa}$ and a consequent decrease by $4 \mathrm{dpa}(n=25$ for 0 -dpa, 1-dpa and 2-dpa samples, and $n=15$ for the 4-dpa data set). The difference is highly significant for all three examined cell types $(P<0.005)$. In U0126-treated animals $(n=25)$ at $2 \mathrm{dpa}$, the amount of autophagic structures is significantly lower $(P<0.0001)$ (Student's $t$-test, error bars refer to S.E.M.)

complete halt of the regeneration process in animals defective for Mapk/Erk activity (Figures $\left.5 a-b^{\prime}\right)$. This effect was comparable to treatments with SU5402, a smallmolecule inhibitor of the Fgf receptor, that also impairs regeneration of surgically removed fins (Figures $\left.5 d-e^{\prime}\right){ }^{10,18}$

The Ras-Mapk/Erk signalling pathway was identified previously as an important stimulator of the autophagic process. ${ }^{34,35}$ Therefore, we decided to test whether the impairment of Mapk/Erk signalling influences autophagic activity in amputated caudal fins. Our electron microscopy analysis revealed reduced numbers of autophagic structures in the distal zone of caudal fins of $2 \mathrm{dpa}$ animals treated with
U0126 (Figure 1g). This implies that Mapk/Erk activity is required for upregulation of autophagy during caudal fin regeneration.

To test this hypothesis further, we took advantage of a transgenic fish line expressing a dominant-negative form of Ras (DNRas) under a heat shock promoter (hs:DNRAS). ${ }^{37}$ Ectopic expression of DNRas impaired regeneration and blastema formation (Figures $5 c$ and $c^{\prime}$ ). We also found that treatment with SU5402 led to basal levels of fluorescence in GFP-Lc3 blastemas (Figures $5 e$ and $e^{\prime}$ ). Similar results were obtained from transgenic animals treated with U0126 (data not shown). 

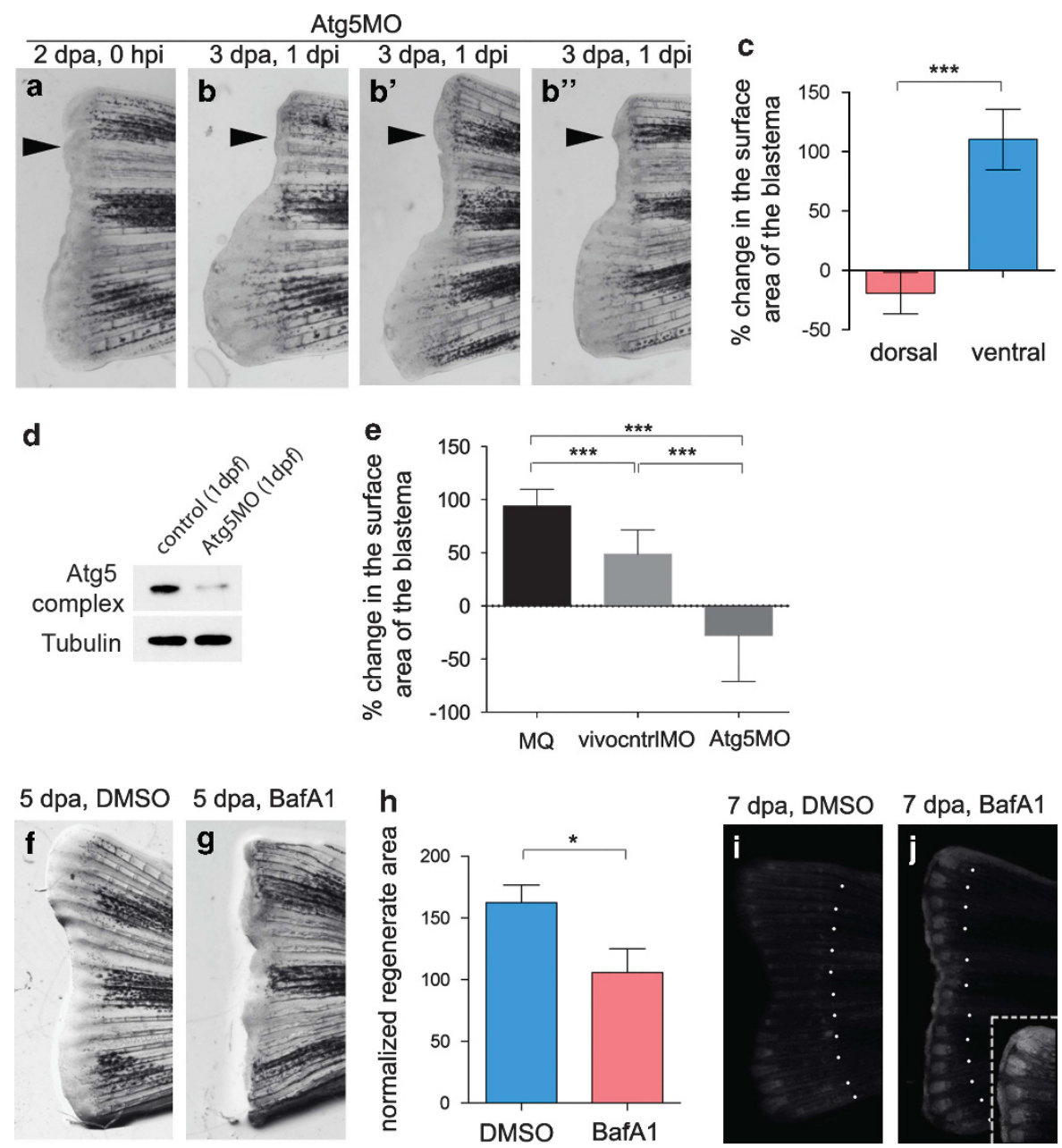

Figure 3 Macroautophagy is required for caudal fin regeneration. (a-c) When Atg5MO was injected into dorsal areas of 2-dpa blastemas, regeneration was severely compromised 1 day after the injection, compared with uninjected ventral fins (black arrowheads indicate site of injection). On panels $\mathbf{b}^{\prime}$ and $\mathbf{b}^{\prime \prime}$, additional examples of $3 \mathrm{dpa}$, 1 day post injection regenerates are shown. Statistical analysis (c) was performed for $n=9$ fins, $P<0.001$ (indicated by ***), paired $t$-test. (d) The efficacy of the Atg5MO was tested in western blots. In 1-dpf embryos the intensity of the band demarking the Atg12-Atg5 complex was reduced to less than 20\%, indicating a potent inhibition of the Atg5 translation. (e) The effectiveness of the Atg5MO in inhibiting regeneration was significant compared with MilliQ water (MQ) and a standard control vivo MO (vivocntrlMO). (Statistical analysis was performed for $n=5$ fins from isogenic wild-type tue background fish for each treatment, $P<0.001$ for each pairwise comparison (indicated by ***), unpaired $t$-test.) (f-h) Regenerating fins were treated with either DMSO (f) or bafilomycin $A_{1}(\mathbf{g})$, a potent inhibitor of autophagosome-autolysosome fusion. The latter treatment significantly impaired regeneration ( $h$ ) at $5 \mathrm{dpa}$ ( $n=6$ fins, $P<0.05$, unpaired $t$-test). (i and $\mathbf{j}$ ) When the fins of GFP-Lc3 transgenic fish were amputated, treatment with bafilomycin $A_{1}$ led to a massive accumulation of the reporter in the blastema (i), as compared with DMSO controls (i). Inset in (j) shows the dorsal area of the representative fin at higher magnification. Dotted lines indicate amputation sites. Dpa, days post amputation; hpi, hours post injection; dpi, days post injection. Error bars refer to S.E.M.

We also examined the expression of the Mapk/Erk signalling marker mkp3 (mitogen-activated protein kinase phosphatase 3) in the blastema, and found that it was not affected in autophagy-defective fish, as compared with normal, non-treated animals (Figures $5 \mathrm{f}-\mathrm{i}$ ). To further confirm that autophagy acts downstream of Mapk/Erk signalling, serum-starved human (HeLa) cells transgenic for a GFP::RFP::Atg8/LC3 reporter were treated with U0126 and analysed with fluorescence microscopy. Untreated cells contained significantly more yellow and red foci (autophagosomes and mature autolysosomes, respectively) than the treated cells (Figures $5 j-I$ ). Together, our data are in accordance with a possible model where autophagy acts downstream of Fgf-Mapk/Erk signalling to mediate cellular reorganisation and promote cell survival during the regeneration of amputated caudal fins in zebrafish (Figure 5m, Supplementary Figure S3).

\section{Discussion}

Most regeneration studies hitherto concentrated on transcriptional events occurring during tissue restoration, yet ultrastructural observations already provided clear evidence that the cytoplasmic component of dedifferentiating cells is dramatically reorganised during heart and caudal fin regeneration in the zebrafish. ${ }^{5,10}$ In this study, we observed increased autophagic activity in the blastema during regeneration of amputated caudal fins (Figure 1), suggesting a previously unaccounted biological role for a lysosomemediated intracellular degradation process in this model of 
Control

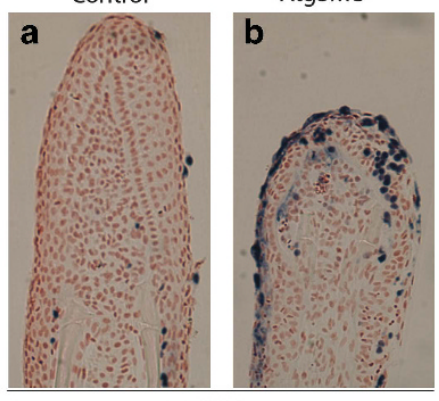

TUNEL

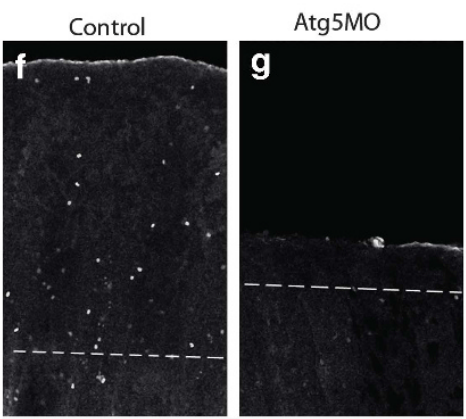

anti-PH3
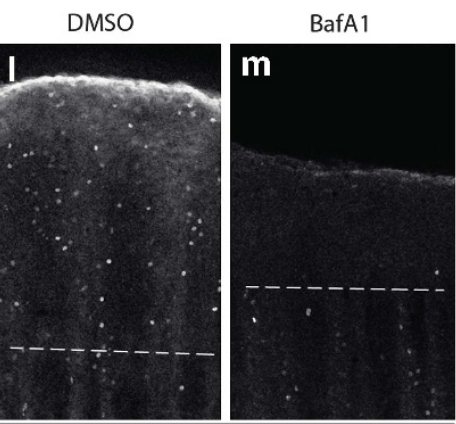

anti-PH3
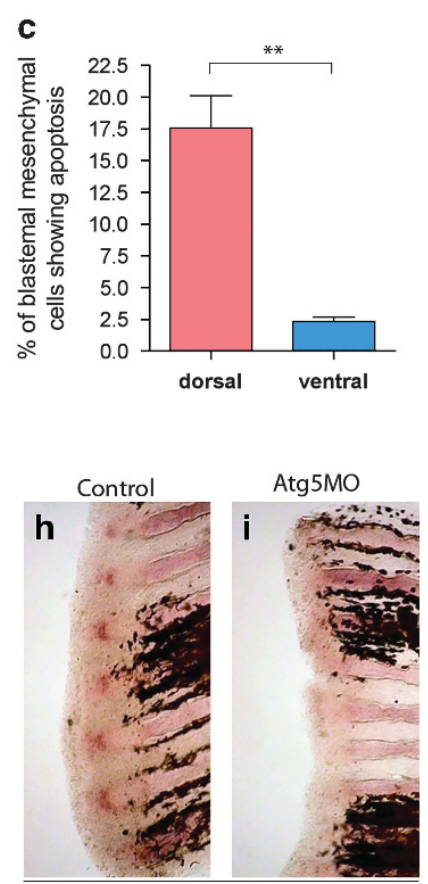

run $\times 2 b$

DMSO
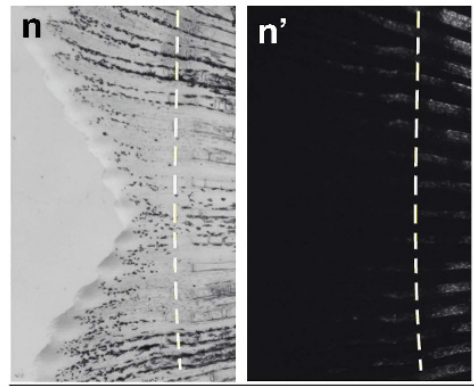

DMSO

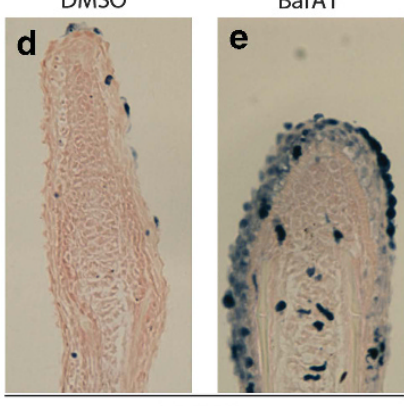

TUNEL

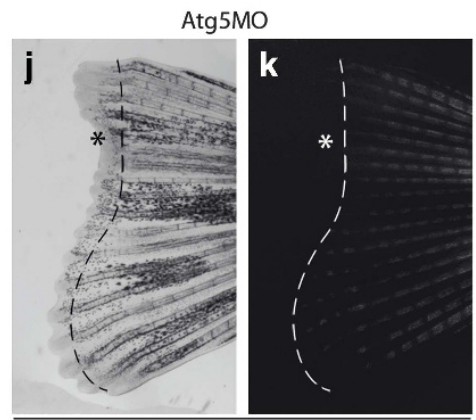

osteocalcin:GFP

BafA1
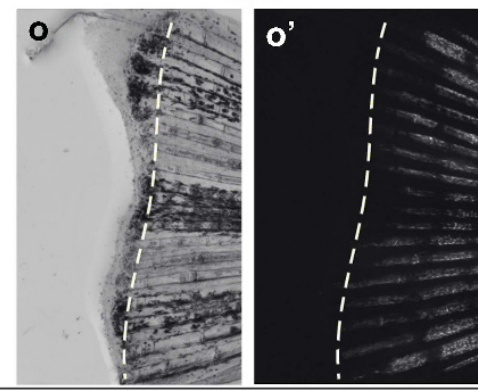

osteocalcin:GFP

Figure 4 Reduced autophagic activity impairs cell survival, proliferation and differentiation during regeneration. (a-c) When dorsal 2-dpa blastema cells were injected with Atg5MO and fixed at $6 \mathrm{hpi}$, TUNEL staining showed increased cell death in the blastemal mesenchyme of treated fin halves, suggesting that autophagy is required for the survival of dedifferentiating cells. (For the statistical analysis apoptotic and non-apoptotic cells were counted on 62 ventral and 49 dorsal sections, ${ }^{\star \star} P<0.01$, paired $t$-test.) (d and e) Chronic treatment with bafilomycin $A_{1}$ also results in reduced regeneration and increased cell death at 2 dpa in the blastemal mesenchyme. (f and $\mathbf{g}$ ) At $3 \mathrm{dpa}$ significant amount of mitotic cells, marked by anti-phospho-histone 3 staining can be detected in a control blastema, whereas the number of proliferating cells is significantly reduced in Atg5MO-injected blastemas. (h and i) Six hours post injection 2-dpa caudal fins were fixed and stained for runx2b expression. Whereas the uninjected ventral half shows runx $2 b$ staining in the blastema at the tip of the individual fin rays (h), in injected dorsal blastema no staining could be observed (i), suggesting that the survival of pre-osteoblasts was impaired upon morpholino treatment. ( $\mathbf{j}$ and $\mathbf{k}$ ) An amputated adult fish shows no osteocalcin:GFP expression at 6 dpa in the Atg5MO-treated dorsal areas. White lines indicate the extent of GFP expression in the bright field (left) and the corresponding fluorescent image (right). The asterisk indicates parts of the blastema tissue that fail to express osteocalcin. (I and $\mathbf{m}$ ) Chronic treatment with bafilomycin $A_{1}$ decreases the number of mitotic cells at $3 \mathrm{dpa}$. ( $\left.\mathbf{n}-\mathbf{o}^{\prime}\right)$ Long-term impairment of autophagic activity with bafilomycin $A_{1}$ suppresses (re)differentiation as shown by the osteocalcin:GFP reporter line in 6 dpa regenerates. Unlike in controls ( $\mathbf{n}^{\prime}$ ), in treated fish no new osteocalcin-positive cells could be observed at $6 \mathrm{dpa}$ in the fin ( $\left.\mathbf{o}^{\prime}\right)$ (Dashed lines denote the plane of amputation.)

Figure 5 Inhibition of the Mapk/Erk signalling pathway blocks regeneration and impairs autophagy induction in the blastema. (a and $\mathbf{b}$ ) Inhibition of Mapk/Erk signalling using U0126 impaired caudal fin regeneration (panels $\mathbf{a}^{\prime}$ and $\mathbf{b}^{\prime}$ are magnifications from the central fin areas of the samples on panels $\mathbf{a}$ and $\mathbf{b}$, respectively). (c and $\mathbf{c}^{\prime}$ ) Inhibition of Ras-MAPK signalling using a transgenic $h s: D N R a s$ reporter line also impaired regeneration and blastema formation. (d and e) Regeneration of caudal fin can be inhibited with SU5402, which also results in a decrease in GFP-LC3 activity. (f-i) mkp3 expression does not depend on autophagic activity. Atg5MO-injected dorsal fin blastemas express $m k p 3$ at levels comparable to that in uninjected ventral fins ( $\mathbf{f}$ and $\mathbf{g}$ ). Similarly, $m k p 3$ staining displays a similar extent of labelling in DMSO-treated control (h) and autophagy-defective, bafilomycin $A_{1}\left(B_{1} A_{1}\right)$-treated backgrounds (i). (j-I) Serum-starved, DMSO-treated GFP-mRFP-LC3 HeLa cells show significant levels of autophagic activity, as denoted by the yellow and red puncta (autophagosomes and autolysosomes, respectively) (j and I). When $10 \mu \mathrm{M}$ or $100 \mu \mathrm{M}$ U0126 was added to the medium, we observed a drop in the autophagic activity ( $\mathbf{k}$ and $\mathbf{~ I ) . ~ A t ~ c o n c e n t r a t i o n s ~ c o m p a r a b l e ~ t o ~ o u r ~ t r e a t m e n t s , ~ t h e ~ e f f e c t ~ o f ~ U 0 1 2 6 ~ w a s ~ h i g h l y ~ s i g n i f i c a n t ~ ( I ) . ~} n=43$ cells measured in both control and treated groups, ${ }^{\star * \star} P<0.001$, unpaired $t$-test, error bars refer to S.E.M. (m) Schematic diagram showing the proposed interaction between the Fgf-Mapk/Erk signalling axis and autophagy. Injury activates Fgf signalling in the fin, which leads to the phosphorylation of Mapk/Erk. Activated Mapk/Erk regulates the sequestration step of autophagy, an essential step in the degradation of the cytosolic component. The small molecule reagents and the MO used in this study inhibit the process at the indicated steps. The three arrows from 'ERK' to 'Autophagy' indicate an indirect link between Mapk/Erk signalling and autophagy 
2 dpa, DMSO

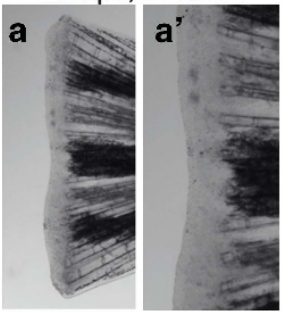

2 dpa, 25 uM U0126
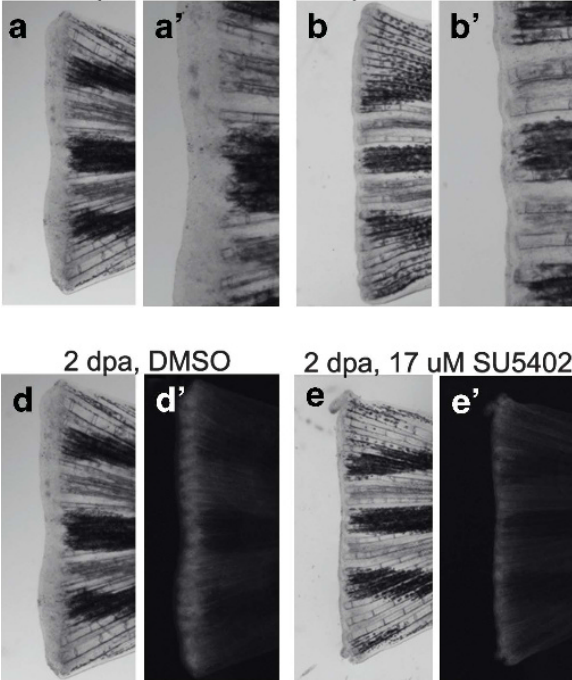

2 dpa, 17 uM SU5402
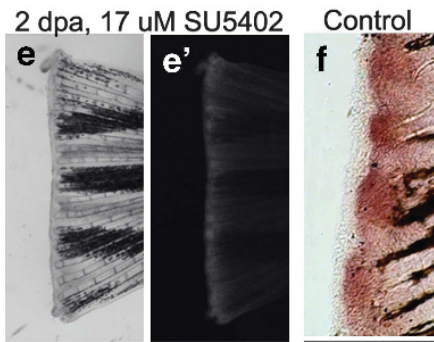

Atg5MO

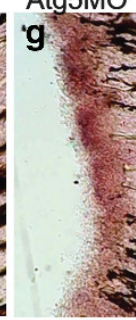

$m k p 3$

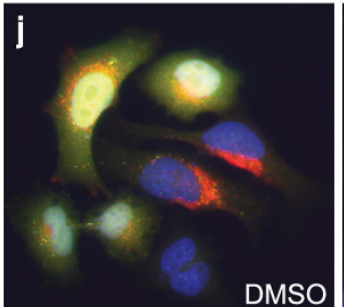

k
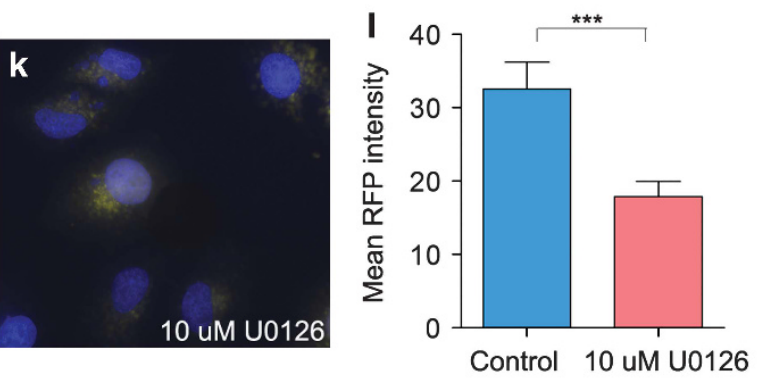

m
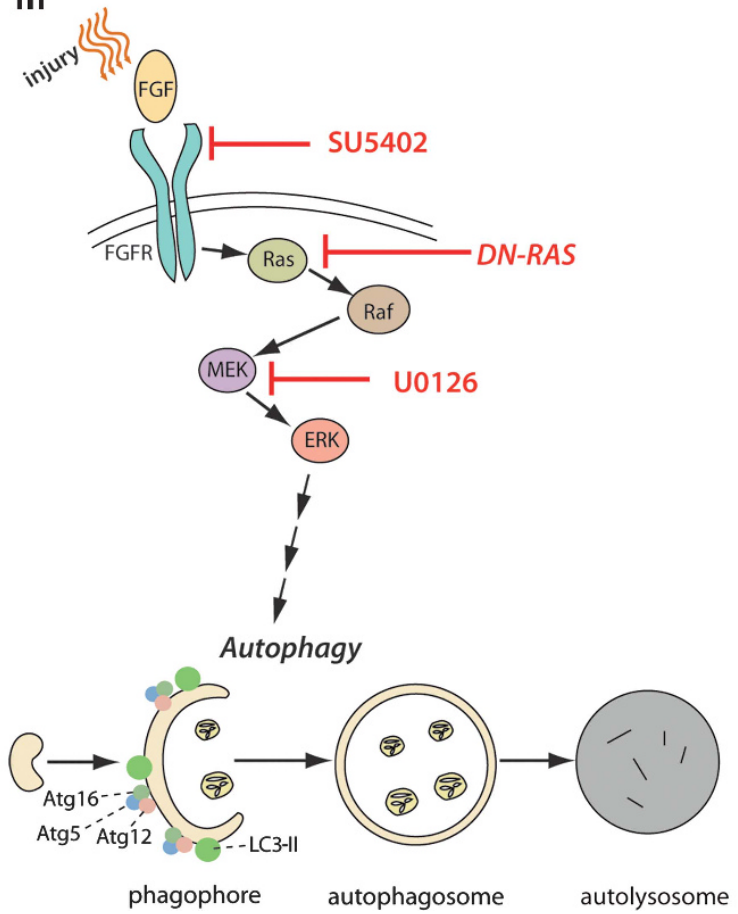
tissue restoration. Supporting the novelty of these results, none of the recent major in-depth reviews of vertebrate regeneration $^{2-4}$ cover autophagy to any extent, clearly showing that the role of this process has been hitherto undervalued, if appreciated at all.

An increase in autophagic activity was also reported during planarian regeneration, ${ }^{38}$ a process reliant on a specialized pool of stem-cell-like cNeoblasts. ${ }^{4}$ However, a general requirement for autophagy in that tissue replacement model was not tested by genetic tools. Therefore, we could not answer the fundamental question of whether upregulation of autophagy was a simple consequence of cellular stress caused by the injury or an underlying causative mechanism of tissue renewal, which may provide energy and building blocks for proliferating cells or remove specific cytoplasmic components to allow cellular differentiation.

In caudal fin blastemas of autophagy-defective zebrafish, significant levels of apoptotic cell death were observed (Figures $3 a-c)$. It is unlikely that such a massive amount of cells with apoptotic features could be generated as a simple consequence of failure in the heterophagic elimination of apoptotic cell corpses, as was suggested earlier. ${ }^{19}$ Thus, our present data further support an active role for autophagy in preventing intensively proliferating and differentiating cells from undergoing apoptosis, similarly to what was shown in mammalian cell lines and nematodes. ${ }^{29,30}$ These results suggest that (re)patterning of amputated caudal fin in zebrafish depends on the death-suppressing function of the autophagic process.

Furthermore, surviving blastema cells from the autophagydefective zebrafish failed to express differentiation markers, such as runx $2 b$ and osteocalcin (Figure 4), ${ }^{10}$ and were eventually unable to regrow surgically removed caudal fins (Figure 3). Thus, autophagic degradation also appears to be required for cellular remodelling that underlies cell fate reprogramming during injury-induced regeneration. Our present study provides the first evidence that autophagy acts as a prerequisite for the (re)formation of a complete organ, the zebrafish caudal fin.

Fgf signalling has been demonstrated to be essential for initiating both zebrafish fin regeneration and homoeostatic regeneration. ${ }^{18,39}$ However, the effector (downstream) mechanisms that mediate Fgf receptor activity in these regeneration processes remain elusive. Previously, the Ras-Mapk/Erk pathway, one of the signalling modules downstream of Fgf receptor activation, has been linked to the regulation of autophagy. There is some ambiguity as to how exactly Mapk/Erk regulates autophagy, but links to BECN1 and G-protein-type regulators of the autophagic machinery have been proposed, ${ }^{40,41}$ as well as a role in the control of autophagosome maturation. ${ }^{34}$ We showed here that a specific inhibitor of Mapk/Erk, which is able to downregulate the autophagic process in other organisms ${ }^{35}$ and in human cells (Figures $5 j-1$ ), effectively blocks fin regeneration in the zebrafish model (Figure 1g, Figures 5a-b'). Furthermore, impairment of Ras activity also inhibits effective regeneration (Figures $5 c$ and $c^{\prime}$ ). Overall, our results support a model, where the activation of the Fgf-Ras-Mapk/Erk signalling axis, following amputation, induces concomitant activation of a conserved genetic dedifferentiation programme and reorganisation of the cytoplasmic milieu in differentiated cells close to the amputation plane. The profound changes in the cytoplasmic component are dependent on autophagy and required for the generation of proliferating unipotent progenitor cells of the blastema (Supplementary Figure S3). Such a potential role for autophagy during regeneration is also highlighted by a recent study, where reprogramming of somatic cells into induced pluripotent stem cells (iPSCs) was significantly enhanced by autophagy-promoting drugs. ${ }^{42}$

The ultimate goal of regenerative medicine is to enable the regrowth of damaged or lost tissues in humans. For example, restoring neurons damaged in an accident, or cardiac muscle lost due to heart attack, has the potential to dramatically increase survival and life quality in the affected individuals. Our present finding that autophagy is required for zebrafish caudal fin regeneration may help to develop innovative strategies to stimulate regeneration by modulating autophagic activity in the damaged tissues. Finally, the importance of autophagic degradation in trauma-induced tissue renewal also raises the possibility that autophagy functions in homoeostatic regeneration, the natural replacement of cells that become lost due to minor damage that occurs every day and during aging. This may reflect a novel aspect of aging control by autophagy. ${ }^{43}$

\section{Materials and Methods}

Fish care. Wild-type fish of the ekwill (ekw) strain and transgenic zebrafish outbred into the same genetic background of 4-12 months of age were used. Fin amputations were performed according to the standard protocols of the field. ${ }^{44}$ After surgery, the fish were kept at $28.5^{\circ} \mathrm{C}$. During heat shock experiments, water temperature was gradually increased to $38^{\circ} \mathrm{C}$. After 40 -min incubation the fish were placed back at $28.5^{\circ} \mathrm{C}$. Heat shocks were delivered after amputation and at 1 and $2 \mathrm{dpa}$, respectively. All fish stocks were maintained in the Animal Facility of Eötvös Loránd University. All protocols used in this study were approved by the Hungarian National Food Chain Safety Office (Permit Number: XIV-I-001/ 515-4/2012).

Electron microscopy. Tail tissues were fixed overnight in $2 \%$ paraformaldehyde (PFA) (Sigma-Aldrich, St. Louis, MO, USA, 158127), 2\% glutaraldehyde (TAAB, G011), $1 \%$ sucrose, $0.028 \% \mathrm{CaCl}_{2}$ (Sigma-Aldrich, C5670) in $0.1 \mathrm{M}$ sodium cacodylate (Sigma-Aldrich, $\mathrm{C0250)}(\mathrm{pH} 7.4)$, then rinsed with cacodylate for 3 days at $4{ }^{\circ} \mathrm{C}$. Samples were post fixed with $0.5 \% \mathrm{OsO}_{4}$ (Sigma-Aldrich, 419494) and embedded in Durcupan (Electron Microscopy Sciences, Hatfield, PA, USA, 14020) plastic resin. Ultrathin sections were collected on formvar-coated grids and stained with lead citrate and uranyl acetate. The samples were observed with a JEOL CM-II 100 electron microscope.

Morpholino experiments. To block the activity of atg5 transcripts, synthetic morpholino antisense oligonucleotides (MOs) were synthesised (GeneTools, Philomath, OR, USA) to mask the translational start site. The Atg5MO sequence was as follows $\left(5^{\prime}\right.$ to $\left.3^{\prime}\right)$ : CCTTGTCATCTGCCATTATCATCGT. The morpholino was vivo-porter coupled, as this system was shown previously to be highly effective in zebrafish balstemas. ${ }^{45}$ MOs were injected into regenerates as described previously $y^{45,46}$ or at the 1-2 cell stage, into the yolk, under the cytoplasm. In the case of the osteocalcin:GFP reporter line, dorsal fin halves were injected every day from $2 \mathrm{dpa}$ on. As a control, Gene Tools' standard vivo control MO was used.

In situ hybridisation. Digoxigenin-labelled antisense probes for run $2 b^{47}$ and $m k 3^{48}$ were synthesised as described previously. ${ }^{49}$ Detection of mRNA transcripts was performed as follows: Fin fragments were fixed in 4\% PFA (PFA; Taab Laboratories Equipment Ltd, Reading, UK) in phosphate-buffered saline containing $0.1 \%$ Tween- 20 (PBST) overnight at $4{ }^{\circ} \mathrm{C}$, washed briefly in PBST and 
then transferred into methanol and stored at $-20^{\circ} \mathrm{C}$. Samples were rehydrated at room temperature through a 10 min wash in $50 \%$ methanol-PBST mix, followed by four 5-min washes in PBST Then, fins were incubated with $10 \mu \mathrm{g} / \mathrm{ml}$ proteinase $\mathrm{K}$ in PBST for $40 \mathrm{~min}$, followed by a 20 -min refixation in $4 \%$ PFA in PBST After five more washes of $5 \mathrm{~min}$ in PBST, samples were prehybridized for $3 \mathrm{~h}$ at $65{ }^{\circ} \mathrm{C}$ in $50 \%$ formamide, $5 x$ SSC, $0.1 \%$ Tween- $20,50 \mu \mathrm{g} / \mathrm{ml}$ heparin and $500 \mu \mathrm{g} / \mathrm{ml}$ torula yeast RNA, adjusted to $\mathrm{pH} 6.0$ with citric acid, and then hybridised overnight in the same buffer including a 1:400 dilution of the digoxigenin-labelled probes. The second day, the samples were rinsed in $75 \%$ formamide/25\% 2x SSC solution, followed by a $1 \mathrm{~h}$ wash in $50 \%$ formamide $/ 50 \% 2 \times$ SSC solution at $65^{\circ} \mathrm{C}$. Two 10 min washes with $2 x$ SSC in PBST followed at room temperature, and a $10 \mathrm{~min}$ wash in $0.1 \mathrm{M} \mathrm{NaCl}, 0.01 \mathrm{M}$ Tris- $\mathrm{HCl}(\mathrm{pH} 8.0), 0.1 \%$ Tween-20 (RNase buffer). Next, samples were incubated for 10 min with $20 \mu \mathrm{g} / \mathrm{ml} \mathrm{RNase} \mathrm{A}$ and $10 \mathrm{U} / \mathrm{ml}$ RNase T1 in RNase buffer, and washed for another 10 min with 2x SSC in PBST Samples were returned to $65^{\circ} \mathrm{C}$ into the $50 \%$ formamide/50\% SSC buffer for $1 \mathrm{~h}$, followed by 15 min washes with $2 x$ SSC in PBST, $0.2 x$ SSC in PBST and PBST, all at room temperature. Samples were incubated for $1 \mathrm{~h}$ in Blocking Reagent (Roche, 11096176001) and with a 1:4000 dilution of an alkaline phosphatase conjugated anti-digoxigenin antibody (Roche Applied Science, Penzberg, Germany, 11093274910) overnight at $4{ }^{\circ} \mathrm{C}$. In the last day, after three rinses with PBST, samples were washed six times for $15 \mathrm{~min}$ in PBST Before detection, three 5-min washes in an alkaline phosphatase buffer (100 mM Tris- $\mathrm{HCl}(\mathrm{pH} 9.5)$, $50 \mathrm{mM} \mathrm{MgCl} 2, \quad 100 \mathrm{mM} \mathrm{NaCl}, \quad 0.1 \%$ Tween-20) followed. $1 \times \mathrm{NBT} / \mathrm{BCIP}$ (combination of nitro blue tetrazolium and 5-bromo-4-chloro-3-indolyl phosphate) substrate was used as chromogen. Samples were kept in the dark during the staining reaction. The reaction was stopped with three brief rinses in PBST, followed by a post-fixation with 4\% PFA in PBST Samples were stored in $80 \%$ glycerol at $4{ }^{\circ} \mathrm{C}$. For imaging, fin fragments were mounted on a standard glass slide.

Western blots. Western blots were performed as described previously. ${ }^{50}$ Anti$\gamma$-tubulin antibody, used at 1:10000 dilution was obtained from Sigma-Aldrich (T5326), and anti-Atg5 antibody, diluted to 1:1000 was purchased from Novus Biologicals (Littleton, CO, USA, NB110-53818). Quantification of the bands was done with ImageJ software (http://rsbweb.nih.gov/ij/).

Immunohistochemistry. Anti-phospho-histone 3 staining on 3 dpa regenerates were performed as described previously. ${ }^{31}$

Cell death assays. TUNEL staining on fin samples was performed using an ApopTag kit (Chemicon/Millpore, Billerica, MA, USA, S7105, S7106, S7107), following the manufacturer's protocol. Samples were fixed, rehydrated, blocked and developed as described for the in situ hybridisation protocol. Samples were embedded in JB-4 resin and sectioned. Sections were counterstained with $0.002 \%$ Neutral Red for $15 \mathrm{~min}$. Slides were subsequently dried and covered. After imaging, cells were counted manually in the blastemal mesenchyme.

Treatments with small molecule reagents. Bafilomycin $A_{1}$ (Sigma-Aldrich, B1793) was dissolved in DMSO to create a $100 \mu \mathrm{M}$ stock solution and then diluted to a concentration of $20 \mathrm{nM}$ in fish water. U0126 (Promega, Fitchburg, WI, USA, V1121) was dissolved in DMSO and then diluted in E3 embryo medium to $25 \mu \mathrm{M}$ final concentration. SU5402 (Merck Millipore, Damstadt, Germany, 572631) was used at $17 \mu \mathrm{M}$ concentration. Control animals (exposed to $0.1 \%$ DMSO) and treated counterparts were always kept at $28.5^{\circ} \mathrm{C}$, with their medium changed daily. The GFP-RFP-LC3 HeLa cell line ${ }^{51}$ was cultured in DMEM (Dulbecco's Modified Eagle's medium; Sigma-Aldrich, D7777) containing $4500 \mathrm{mg} / \mathrm{l}$ glucose, $10 \%$ heat-inactivated fetal calf serum (Merck, Damstadt, Germany), $50 \mu \mathrm{g} / \mathrm{ml}$ gentamycin (Hungaropharma, Budapest, Hungary) and $600 \mu \mathrm{g} / \mathrm{ml} \mathrm{G} 418$ (Sigma-Aldrich, G8168). Cells $\left(2 \times 10^{4}\right)$ were plated onto 10-mm diameter poly-D-lysine coated coverslips in 24-well plates (Greiner Bio-One, Frickenhausen, Germany) $10 \mathrm{~h}$ before the treatment. Autophagy was induced by serum starvation in fetal calf serum-free medium while cells were exposed to $10 \mu \mathrm{M}$ U0126 or $0.1 \%$ DMSO as control for $14 \mathrm{~h}$. Cells were fixed in $4 \%$ PFA and mounted in bis-benzimide (Sigma-Aldrich) containing mowiol 4.88 (Polysciences, Warrington, PA, USA). Epifluorescent images were taken of each condition using a BX51 microscope (Olympus, Tokyo, Japan) fitted with a FluoViewll camera and the AnalysisPro software (Olympus), using a $\times 60 / 1.4$ oil Plan objective and the appropriate filter sets (DAPI: BP330-385/DM400/BA420; GFP: BP460-500/ DM505/BP510-560; RFP: BP480-550/DM570/BA590). Images were analysed by
ImageJ software. The quantity of RFP-positive autolysosomes (autophagosomes processed in the degradation pathway) was compared by measuring the RFP mean intensity in U0126-treated and control cells.

Imaging, fin area measurements and analysis. Bright field and fluorescence pictures of live fin regenerates were taken with a Zeiss AxioZoom stereomicroscope. Whole mount in situ hybridisation pictures were taken with a Nikon E1000 microscope. Changes in the size of regenerates were calculated using pictures taken right after injection and 1 day post injection. Relative areas of dorsal and ventral regenerates were calculated using the ImageJ software. The same programme was used to calculate regenerate sizes of DMSO- and bafilomycin $\mathrm{A}_{1}$-treated fins, where normalisation was done by dividing the area of the regenerate by the width of the fin. Statistical analysis (paired and unpaired $t$-test) was performed using the Prism (GraphPad) software.

\section{Conflict of Interest}

The authors declare no conflict of interest.

Acknowledgements. This work is supported by the Hungarian Scientific Research Funds NK78012 and K109349. MV, KT-V and TV are grantees of the János Bolyai scholarship. DJK is supported by NIH grant GM053396. We thank Steve Wilson and members of the Wilson lab for technical help, Gilbert Weidinger and Alex Nechiporuk for the osteocalcin:GFP and hs:DNRas lines, David Rubinsztein for the GFP::RFP::Atg8/LC3 HeLa cell line and Kara Cerveny and Julianna Vig for helpful comments and suggestions regarding the manuscript.

1. Berthiaume F, Maguire TJ, Yarmush ML. Tissue engineering and regenerative medicine: history, progress, and challenges. Annu Rev Chem Biomol Eng 2011; 2: 403-430.

2. Jopling C, Boue S, Izpisua Belmonte JC. Dedifferentiation, transdifferentiation and reprogramming: three routes to regeneration. Nat Rev Mol Cell Biol 2011; 12: 79-89.

3. Poss KD. Advances in understanding tissue regenerative capacity and mechanisms in animals. Nat Rev Genet 2010; 11: 710-722.

4. Tanaka EM, Reddien PW. The cellular basis for animal regeneration. Dev Cell 2011; 21: 172-185

5. Jopling C, Sleep E, Raya M, Martí M, Raya A, Izpisúa Belmonte JC et al. Zebrafish heart regeneration occurs by cardiomyocyte dedifferentiation and proliferation. Nature 2010; 464: 606-609.

6. Kikuchi K, Holdway JE, Werdich AA, Anderson RM, Fang Y, Egnaczyk GF et al. Primary contribution to zebrafish heart regeneration by gata $4^{+}$cardiomyocytes. Nature 2010; 464: 601-605

7. Azevedo AS, Sousa S, Jacinto A, Saude L. An amputation resets positional information to a proximal identity in the regenerating zebrafish caudal fin. BMC Dev Biol 2012; 12: 24.

8. Itou J, Kawakami H, Burgoyne T, Kawakami Y. Life-long preservation of the regenerative capacity in the fin and heart in zebrafish. Biology Open 2012; 1: 739-746.

9. Kragl M, Knapp D, Nacu E, Khattak S, Maden M, Epperlein HH et al. Cells keep a memory of their tissue origin during axolotl limb regeneration. Nature 2009; 460: 60-65.

10. Knopf F, Hammond C, Chekuru A, Kurth T, Hans S, Weber CW et al. Bone regenerates via dedifferentiation of osteoblasts in the zebrafish fin. Dev Cell 2011; 20: 713-724.

11. Sousa S, Afonso N, Bensimon-Brito A, Fonseca M, Simões M, Leon J et al. Differentiated skeletal cells contribute to blastema formation during zebrafish fin regeneration. Development 2011; 138: 3897-3905.

12. Tu S, Johnson SL. Clonal analyses reveal roles of organ founding stem cells, melanocyte stem cells and melanoblasts in establishment, growth and regeneration of the adult zebrafish fin. Development 2011; 137: 3931-3939.

13. Singh SP, Holdway JE, Poss KD. Regeneration of amputated zebrafish fin rays from de novo osteoblasts. Dev Cell 2012; 22: 879-886.

14. Blum N, Begemann G. Retinoic acid signaling controls the formation, proliferation and survival of the blastema during adult zebrafish fin regeneration. Development 2012; 139: 107-116.

15. Laforest L, Brown CW, Poleo G, Géraudie J, Tada M, Ekker M et al. Involvement of the sonic hedgehog, patched 1 and bmp2 genes in patterning of the zebrafish dermal fin rays. Development 1998; 125: 4175-4184.

16. Smith A, Avaron F, Guay D, Padhi BK, Akimenko MA. Inhibition of BMP signaling during zebrafish fin regeneration disrupts fin growth and scleroblasts differentiation and function. Dev Biol 2006; 299: 438-454

17. Whitehead GG, Makino S, Lien CL, Keating MT. fgf20 is essential for initiating zebrafish fin regeneration. Science 2005; 310: 1957-1960.

18. Poss KD, Shen J, Nechiporuk A, McMahon G, Thisse B, Thisse C et al. Roles for Fgf signaling during zebrafish fin regeneration. Dev Biol 2000; 222: 347-358.

19. Mizushima N, Levine B, Cuervo AM, Klionsky DJ. Autophagy fights disease through cellular self-digestion. Nature 2008; 451: 1069-1075. 
20. Levine B, Klionsky DJ. Development by self-digestion: molecular mechanisms and biological functions of autophagy. Dev Cell 2004; 6: 463-477.

21. Mizushima N, Levine B. Autophagy in mammalian development and differentiation. Nat Cell Biol 2010; 12: 823-830.

22. Klionsky DJ et al. Guidelines for the use and interpretation of assays for monitoring autophagy in higher eukaryotes. Autophagy 2008; 4: 151-175.

23. He C, Bartholomew CR, Zhou W, Klionsky DJ. Assaying autophagic activity in transgenic GFP-LC3 and GFP-Gabarap zebrafish embryos. Autophagy 2009; 5: 520-526.

24. Klionsky DJ et al. Guidelines for the use and interpretation of assays for monitoring autophagy. Autophagy 2012; 8: 445-544.

25. Chen Y, Klionsky DJ. The regulation of autophagy - unanswered questions. J Cell Sci 2011; 124: 161-170.

26. Saxena S, Singh SK, Lakshmi MG, Meghah V, Bhatti B, Swamy CV et al. Proteomic analysis of zebrafish caudal fin regeneration. Mol Cell Proteomics 2012; 11: 014118

27. Hu Z, Zhang J, Zhang Q. Expression pattern and functions of autophagy-related gene atg5 in zebrafish organogenesis. Autophagy 2011; 7: 1514-1527.

28. Hundeshagen P, Hamacher-Brady A, Eils R, Brady NR. Concurrent detection of autolysosome formation and lysosomal degradation by flow cytometry in a high-content screen for inducers of autophagy. BMC Biol 2011; 9: 38.

29. Boya P, González-Polo RA, Casares N, Perfettini JL, Dessen P, Larochette N et al. Inhibition of macroautophagy triggers apoptosis. Mol Cell Biol 2005; 25: 1025-1040.

30. Takacs-Vellai K, Vellai T, Puoti A, Passannante M, Wicky C, Streit A et al. Inactivation of the autophagy gene bec-1 triggers apoptotic cell death in C. elegans. Curr Biol 2005; 15: 1513-1517.

31. Poss KD, Nechiporuk A, Hillam AM, Johnson SL, Keating MT. Mps1 defines a proximal blastemal proliferative compartment essential for zebrafish fin regeneration. Development 2002; 129: 5141-5149.

32. Lee Y, Grill S, Sanchez A, Murphy-Ryan M, Poss KD. Fgf signaling instructs positiondependent growth rate during zebrafish fin regeneration. Development 2005; 132 5173-5183.

33. Dailey L, Ambrosetti D, Mansukhani A, Basilico C. Mechanisms underlying differential responses to FGF signaling. Cytokine Growth Factor Rev 2005; 16: 233-247.

34. Corcelle E, Djerbi N, Mari M, Nebout M, Fiorini C, Fénichel P et al. Control of the autophagy maturation step by the MAPK ERK and p38: lessons from environmental carcinogens. Autophagy 2007; 3: 57-59.

35. Kang C, You YJ, Avery L. Dual roles of autophagy in the survival of Caenorhabditis elegans during starvation. Genes Dev 2007; 21: 2161-2171.
36. Favata MF, Horiuchi KY, Manos EJ, Daulerio AJ, Stradley DA, Feeser WS et al. Identification of a novel inhibitor of mitogen-activated protein kinase kinase. J Biol Chem 1998; 273: 18623-18632.

37. Lee Y, Lee Y, Hami D, De Val S, Kagermeier-Schenk B, Wills AA et al. Maintenance of blastemal proliferation by functionally diverse epidermis in regenerating zebrafish fins. Dev Biol 2009; 331: 270-280.

38. Gonzalez-Estevez C, Felix DA, Aboobaker AA, Salo E. Gtdap-1 promotes autophagy and is required for planarian remodeling during regeneration and starvation. Proc Natl Acad Sci USA 2007; 104: 13373-13378.

39. Wills AA, Kidd AR III, Lepilina A, Poss KD. Fgfs control homeostatic regeneration in adult zebrafish fins. Development 2008; 135: 3063-3070.

40. Wang J, Whiteman MW, Lian H, Wang G, Singh A, Huang D et al. A non-canonical MEK/ERK signaling pathway regulates autophagy via regulating Beclin 1. J Biol Chem 2009; 284: 21412-21424

41. Ogier-Denis E, Pattingre S, El Benna J, Codongo P. Erk1/2-dependent phosphorylation of $\mathrm{G} \alpha$-interacting protein stimulates its GTPase accelerating activity and autophagy in human colon cancer cells. J Biol Chem 2000; 275: 39090-39095.

42. Chen T, Shen L, Yu J, Wan H, Guo A, Chen J et al. Rapamycin and other longevitypromoting compounds enhance the generation of mouse induced pluripotent stem cells Aging Cell 2011; 10: 909-911.

43. Vellai T, Takács-Vellai K, Sass M, Klionsky DJ. The regulation of aging: does autophagy underlie longevity? Trends Cell Biol 2009; 19: 487-494.

44. Poss KD, Shen J, Keating MT. Induction of lef1 during zebrafish fin regeneration. Dev Dyn 2000; 219: 282-286

45. Chablais F, Jazwinska A. IGF signaling between blastema and wound epidermis is required for fin regeneration. Development 2010; 137: 871-879.

46. Hyde DR, Godwin AR, Thummel R. In vivo electroporation of morpholinos into the regenerating adult zebrafish tail fin. $J$ Vis Exp 2012; 29: e3632.

47. Li N, Felber K, Elks P, Croucher P, Roehl HH. Tracking gene expression during zebrafish osteoblast differentiation. Dev Dyn 2009; 238: 459-466.

48. Tsang M, Maegawa S, Kiang A, Habas R, Weinberg E, Dawid IB et al. A role for MKP3 in axial patterning of the zebrafish embryo. Development 2004; 131: 2769-2779.

49. Maegawa S, Varga M, Weinberg ES. FGF signaling is required for $\beta$-catenin-mediated induction of the zebrafish organizer. Development 2006; 133: 3265-3276.

50. Link V, Shevchenko A, Heisenberg CP. Proteomics of early zebrafish embryos. BMC Dev Biol 2006; 6: 1

51. Settembre C, Di Malta C, Polito VA, Garcia Arencibia M, Vetrini F, Erdin S et al. TFEB links autophagy to lysosomal biogenesis. Science 2011; 332: 1429-1433.

Supplementary Information accompanies this paper on Cell Death and Differentiation website (http://www.nature.com/cdd) 